\title{
Impact of number of channels on signal transmission in elastic optical network
}

Indayara B. Martins, G. Pérez-Sánchez, Ivan Aldaya, André A. Costa, F. Rudge Barbosa

Indayara B. Martins, G. Pérez-Sánchez, Ivan Aldaya, André A. Costa, F. Rudge Barbosa, "Impact of number of channels on signal transmission in elastic optical network," Proc. SPIE 10560, Metro and Data Center Optical Networks and Short-Reach Links, 105600A (30 January 2018); doi: 10.1117/12.2290964

SPIE. Event: SPIE OPTO, 2018, San Francisco, California, United States 


\title{
Impact of Number of Channels on Signal Transmission in Elastic Optical Networks
}

\author{
Indayara B. Martins ${ }^{a^{*}}$, G. Pérez-Sánchez ${ }^{\mathrm{b}}$, Ivan Aldaya ${ }^{\mathrm{c}}$, André A.Costa $^{\mathrm{d}}$ F. Rudge Barbosa ${ }^{\mathrm{e}}$ \\ ${ }^{a}$ Pontificia Universidade Catolica de Campinas - CEATEC, PUC-Campinas SP, Brazil \\ ${ }^{\mathrm{b}}$ Universidad Autonoma Metropolitana, Unidad Azcapotzalco, Mexico \\ ${ }^{\mathrm{c}}$ Universidade Estadual Paulista - UNESP, São João da Boa Vista, SP - Brazil \\ ${ }^{d}$ Universidade Federal de Uberlândia - FEELT, Patos de Minas, MG, Brazil \\ ${ }^{\mathrm{e}}$ Universidade de Campinas- FEEC, UNICAMP- Campinas, SP, Brazil \\ * indayara.martins@puc-campinas.edu.br; rudge@dsif.fee.unicamp.br
}

\begin{abstract}
In this paper, the impact of the number of channels on the performance of elastic optical networks (EONs) is examined considering a multilevel modulation format and coherent transmission. Network design parameters such as spectral bandwidth and channel symbol error rate (SER), are analysed. We simulated the transmission of quadrature phase shift-keying (QPSK) signals, modulated at 56 and $100 \mathrm{Gbps}$, to evaluate a proposed flexible spectral allocation method in order to evaluate the effect of number of channels and the required total spectral bandwidth.
\end{abstract}

Keywords: optical channels, flexible spectrum allocation, signal band, symbol error rate, Elastic optical networks

\section{INTRODUCTION}

THE ongoing increase of services and applications such as online video, VoIP, online game, Digital TV, and emergent major applications such as social networks, Internet of things as well as the convergence of wireless and optical wireline has resulted in an exponential growth of Internet date traffic, which has distinct bandwidth demands. According to research conducted by a major supplier [1], by 2020 the global Internet traffic should reach 2.3 ZB (zettabytes = one billion gigabytes); that is, IP traffic will then have increased almost 100 times between 2005 and 2020. This scenario presents challenges for network design engineers both now and in the future, having to face ever-increasing capacity demands, with additional constraints on reliability, flexibility, cost, and low energy consumption

Nowadays optical networks interconnect worldwide multiple locations with variable capacities and transmission rates using Wavelength Division Multiplexing (WDM) systems [2] with up to a hundred channels, each carrying data rates from 10 to $400 \mathrm{Gbps}$, leading to impressive Tb/s aggregates [3].

Traditionally, signal spectra in WDM optical network are allocated as frequency fixed-grid according to ITU$\mathrm{T}$ (International Telecommunication Union, Telecommunication Standardization Sector) standard [4]. The proposal of an Elastic Optical Network architecture supports bandwidth-variable signals (which can have large or small bandwidths), has been identified as a good solution to save spectral band and allows the possibility of transmission rates above $400 \mathrm{Gbps}$, compared with the traditional wavelength division multiplexing (WDM) allocation model established by ITU-T $(100,50,25$ or $12.5 \mathrm{GHz})$ [3], [5-7]. Evidently, the use of EON brings the benefit of the reduction of spectral consumption; however, the quality of signal transmission (Qo T) should be investigated, due to several limitations and penalties of the physical layer [8].

The main goal in this work is to analyze some key network design parameters regarding the signal SER and signal band allocation. Thus is examined the impact of the number of channels on the elastic optical networks 
(EONs) considering the multilevel modulation format and coherent transmission. Analytic calculations and simulations were realized to evaluate the impact that the amount of channels in EON causes on the spectral consumption and on the signal QoT. The first step of results is based in calculations that account the reduction of spectrum consumed comparing with WDM network that adopt grid standard defined for ITU. In the second step, we evaluate through simulation the spectral allocation method in EONs seeking to analyze the effect of the number of channels and required spectral bandwidth. In order to analyze the influence of the physic effects, the spectral bandwidth was analyzed to monitor for the channel symbol error rate (SER). Simulation of the physical layer was configured with variable number of channels between 1 and 31, signals modulated using QPSK, the transmission rates per channel were 56 and $100 \mathrm{Gbps}$, Erbium Fiber amplifiers (EDFA) and dispersion compensating fiber (DCF) were considered to compensate attenuation and dispersion. Fiber spans were configured to $100 \mathrm{~km}$ using standard single mode fiber (SMF). Some non-linear effects were also considered in the simulation set up. Analytic modeling and computer simulations were carried out using VPI ${ }^{\circledR}$ Transmission System, and Matlab®.

\section{BASIC CONCEPTS OF SPECTRUM ALLOCATION}

In this section, some basic concepts and calculations about spectral allocation as a function of the modulation format and transmission rate are followed [9] and presented here.

For the spectrum allocation, the occupied signal bandwidth $\left(\Delta f_{s}\right)$ must related to its transmission rate $(T R)$ and the type of modulation. To allocate a signal inside a spectral grid (fix or elastic), a guard band ( $\left.\Delta f_{G B}\right)$ must be considered, thus the total signal spectral band is given by equation (1):

$$
\Delta f_{s}=\frac{1}{\log _{2} M} * T R+\Delta f_{G B}
$$

where $M$ means number of symbols, characteristic of each modulation format.

To multichannel systems, the calculation of the total spectrum allocation shall be done separately for each channel independently, and in order it is obtained by the sum involving all signals as:

$$
\Delta f_{s t}=\sum_{i=1}^{N} \frac{1}{\log _{2} M_{i}} * T R_{i}+\Delta f_{G B}
$$

where index $i$ represents a specific channel and $N$ is equal to the total number of channels in the system.

The spectrum consumption optimization can be achieved with the signal allocation in finer s pectrum slots, thus to obtain the required slots $(R S)$ is:

$$
R S=\frac{\sum_{i=1}^{N} \frac{1}{\log _{2} M_{i}} * T R_{i}+\Delta f_{G B}}{G S}
$$

where $G S$ is a given grid size.

In other words, the allocated bandwidth should always be larger than the signal bandwidth, including guard bands to compensate physical impairments and to avoid eventual crosstalk. The minimum allocated bandwidth can be calculated by dividing the transmission bit rate by the number of bits per symbol specific of each signal modulation, and then multiplied by two, since this minimum allocated band has to be twice the signal bandwidth [10]. To illustrate what will be used in Table 1, a transmitted signal of $56 \mathrm{~Gb} / \mathrm{s}$ and modulated in quadrature phase shift keying (QPSK) holds 2 bits per symbol and equals 28 Gbaud (56/2), then the minimum allocated signal bandwidth equals $56 \mathrm{GHz}$ (without guard-band). The spectral efficiency is another parameter related to the spectrum utilization and is defined as the ratio of data rate and occupied spectral band, and quantified in units of bits per second per Hertz (b/s/Hz). It means how much the allowed grid space is effectively being used (e.g., 56 $\mathrm{Gb} / \mathrm{s}$ QPSK, allocated in a fixed grid of $75(3 \times 25) \mathrm{GHz}$, will have a spectral efficiency of $0.7 \mathrm{bits} / \mathrm{s} / \mathrm{Hz}$; which is rather low).

Table 1 presents the theoretical values of bandwidth requirement and allocation considering grids of 100 , 50, $25 \mathrm{GHz}$ (ITU standard) and slots of $12.5 \mathrm{GHz}$ (Flex Grid) [3,4], transmission rate of 56 and $100 \mathrm{Gbps}$ and modulation format QPSK, 2 bits by symbol. Through this analysis it can be observed that the (fixed) WDM ITU 
grid slots waste much more bandwidth than the required for the signal to be allocated, because it is $n x$ (slot), where $n$ is the number of slots. The parameter wasted band (Wb1; Wb2, Wb3, Wb4) denotes the wasted bandwidth in the fixed grids and Flex. It is interesting to note that for $56 \mathrm{~Gb} / \mathrm{s}$ signal much is gained in this procedure, but for $100 \mathrm{~Gb} / \mathrm{s}$ (which is exactly $4 \times 25$ and $2 \times 50$ ) the procedure is not necessary. Furthermore, we point out that these results consider only the amount of bandwidth necessary to insert the signal band in the spectrum band, without considering eventual linear and propagation effects in the optical fiber.

\begin{tabular}{|c|c|c|c|c|c|c|c|c|c|}
\hline \multirow{3}{*}{$\begin{array}{c}\text { TR } \\
\text { (Gbps) }\end{array}$} & \multirow{3}{*}{$\begin{array}{c}\text { No. } \\
\text { Channels }\end{array}$} & \multicolumn{8}{|c|}{$\Delta \mathbf{f}_{\text {st }}(\mathbf{G H z})$} \\
\hline & & \multicolumn{6}{|c|}{ ITU Grid } & \multicolumn{2}{|l|}{ Flex Grid } \\
\hline & & $n_{1} \times 25$ & Wb1 & $n_{2} \times 50$ & Wb2 & $n_{3} \times 100$ & Wb3 & $n_{4} \times 12,5$ & Wb4 \\
\hline \multirow[t]{5}{*}{56} & 1 & $3 * 25=75$ & 19 & $2 * 50=100$ & 44 & $1 * 100=100$ & 44 & $5^{*} 12,5=62,5$ & 6,5 \\
\hline & 3 & $7 * 25=175$ & 7 & $4 * 50=200$ & 32 & $2 * 100=200$ & 32 & $14 * 12,5=175$ & 7,0 \\
\hline & 9 & $21 * 25=525$ & 21 & $11 * 50=550$ & 46 & $6 * 100=600$ & 96 & $41 * 12,5=512,5$ & 8,5 \\
\hline & 15 & $34 * 25=850$ & 10 & $17 * 50=850$ & 10 & $9 * 100=900$ & 60 & $68 * 12,5=850$ & 10 \\
\hline & 31 & $70 * 25=1750$ & 14 & $35 * 25=1750$ & 14 & $18 * 100=1800$ & 64 & $139 * 12,5=1737,5$ & 1,5 \\
\hline \multirow[t]{5}{*}{100} & 1 & $4 * 25=100$ & 0 & $2 * 50=100$ & 0 & $1 * 100=100$ & 0 & $8 * 12,5=100$ & 0 \\
\hline & 3 & $12 * 25=300$ & 0 & $6 * 50=300$ & 0 & $3 * 100=300$ & 0 & $24 * 12,5=300$ & 0 \\
\hline & 9 & $36 * 25=900$ & 0 & $18 * 50=900$ & 0 & $9 * 100=900$ & 0 & $72 * 12,5=900$ & 0 \\
\hline & 15 & $60 * 25=1500$ & 0 & $30 * 50=1500$ & 0 & $15 * 100=1500$ & 0 & $120 * 12,5=1500$ & 0 \\
\hline & 31 & $124 * 25=3100$ & 0 & $62 * 50=3100$ & 0 & $31 * 100=3100$ & 0 & $248^{*} 12,5=3100$ & 0 \\
\hline
\end{tabular}

Table 1 - Bandwidth requirement and allocation (theoretical values).

\section{SIMULATION MODEL}

This section describes the network simulation set-up. Simulation of the physical layer is configured with 1, 3, 15, and 31 and channels using QPSK modulation format (see Fig.2). The transmission rates per channel are 56 and 100 Gbps. Erbium Doped Fiber amplifiers (EDFA) and dispersion compensating fiber (DCF) are considered. Spans of $100 \mathrm{~km}$ using standard single mode fiber (SSMF), with attenuation coefficient of $0.2 \mathrm{~dB} / \mathrm{km}$ and dispersion of $16 \mathrm{ps} / \mathrm{km} / \mathrm{nm}$. To DCF has considered an attenuation of $0.5 \mathrm{~dB} / \mathrm{km}$ and dispersion of $-90 \mathrm{ps} / \mathrm{km} / \mathrm{nm}$, as it is presented in the parameters table in Fig.2. All no-linear effects were considered in the simulation. The SER was evaluated on the central channel.

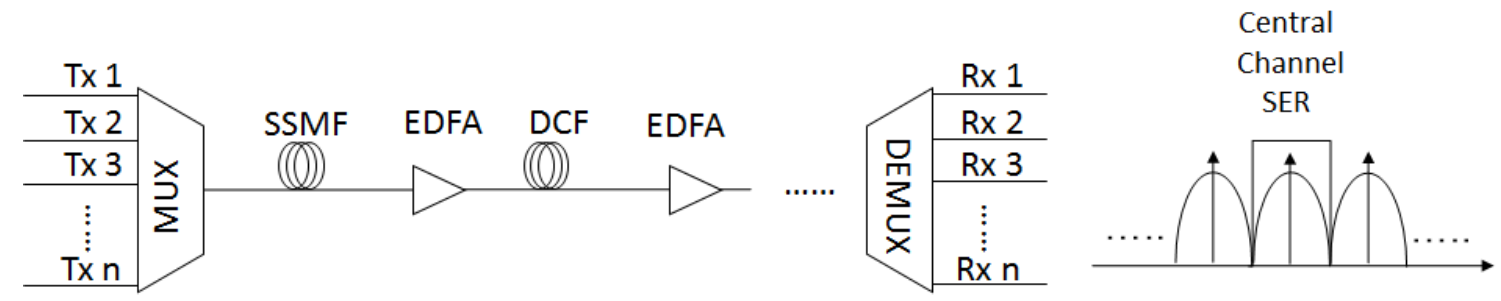

\begin{tabular}{|c|c|c|}
\hline Parameters & $\begin{array}{c}\text { Fiber } \\
(\mathrm{SMF})\end{array}$ & DCF \\
\hline $\mathrm{D}(\mathrm{ps} / \mathrm{nm} / \mathrm{km}) @ 1550 \mathrm{~nm}$, & 16 & -90 \\
\hline Loss, dB/km & 0.2 & 0.5 \\
\hline Fiber link length -km & 100 & 20 \\
\hline D. Slope, $\mathrm{ps} / \mathrm{nm}^{2} / \mathrm{km}$ & 0.08 & -0.45 \\
\hline Effective area, $\mu \mathrm{m}^{2}$ & 80 & 50 \\
\hline Group Refractive Index & \multicolumn{2}{|c|}{1.47} \\
\hline
\end{tabular}

Fig. 1: Schematic diagram of the system simulations and system fiber parameters. 


\section{RESUULTS AND DISCUSSION}

Results show the impact of spectrum allocation as a function of the signal SER and distance. The s ystem uses single channels and multi-channels with the same transmission rate, performance of the central channel are the one presented.

Results in the Fig. 2 show the SER against channel spectral width on the system transmitter (back-to-back transmission) for 56 and $100 \mathrm{Gbps}$. We can see that single channel transmission requires average bandwidth of 25 and $45 \mathrm{GHz}$ for 56 e $100 \mathrm{Gbps}$ signals, respectively. For multichannel systems, 56 and $100 \mathrm{~Gb}$ s will require 40 and $75 \mathrm{GHz}$ respectively.

In Fig. 3 (a \& b), show the SER against channel spectral width on the system considering the fiber links of 100 and $300 \mathrm{Km}$. Note that the signal quality improves with the transmission distance and also between single and multi-channels. The shortest the distance and single channel the smaller the required bandwidth, i.e., lower the number of slots. Also, note that for longer transmission distances and multi channels the required bandwidth for a $\mathrm{SER}=10^{-9}$ increases.

\section{CONCLUSIONS}

In this work were analyzed the minimum signal bandwidth to transmit a multilevel modulation format and multi-channels with high transmission rate on EON. Analytical results based in calculations that account the reduction of spectrum consumed comparing with WDM network that adopt grid standard defined by ITU, was realized and indicate that the ITU fixed slots grids offer much more bandwidth that the required for the signal to be allocated. The results of simulations obtained indicate that the number of channels and the distance affect the SER of the signal due mainly to the physical layer impairments.

For future work, we will be investigate with more details the impact of each physical impairments in optical links.

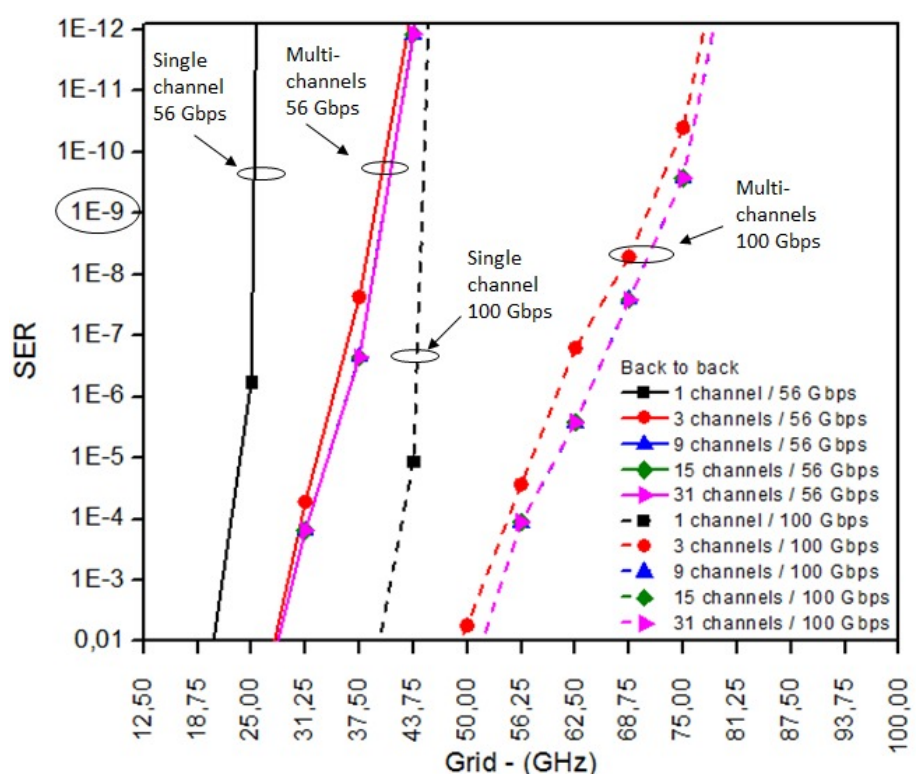

Figure 2: Back to back transmitter - QPSK 

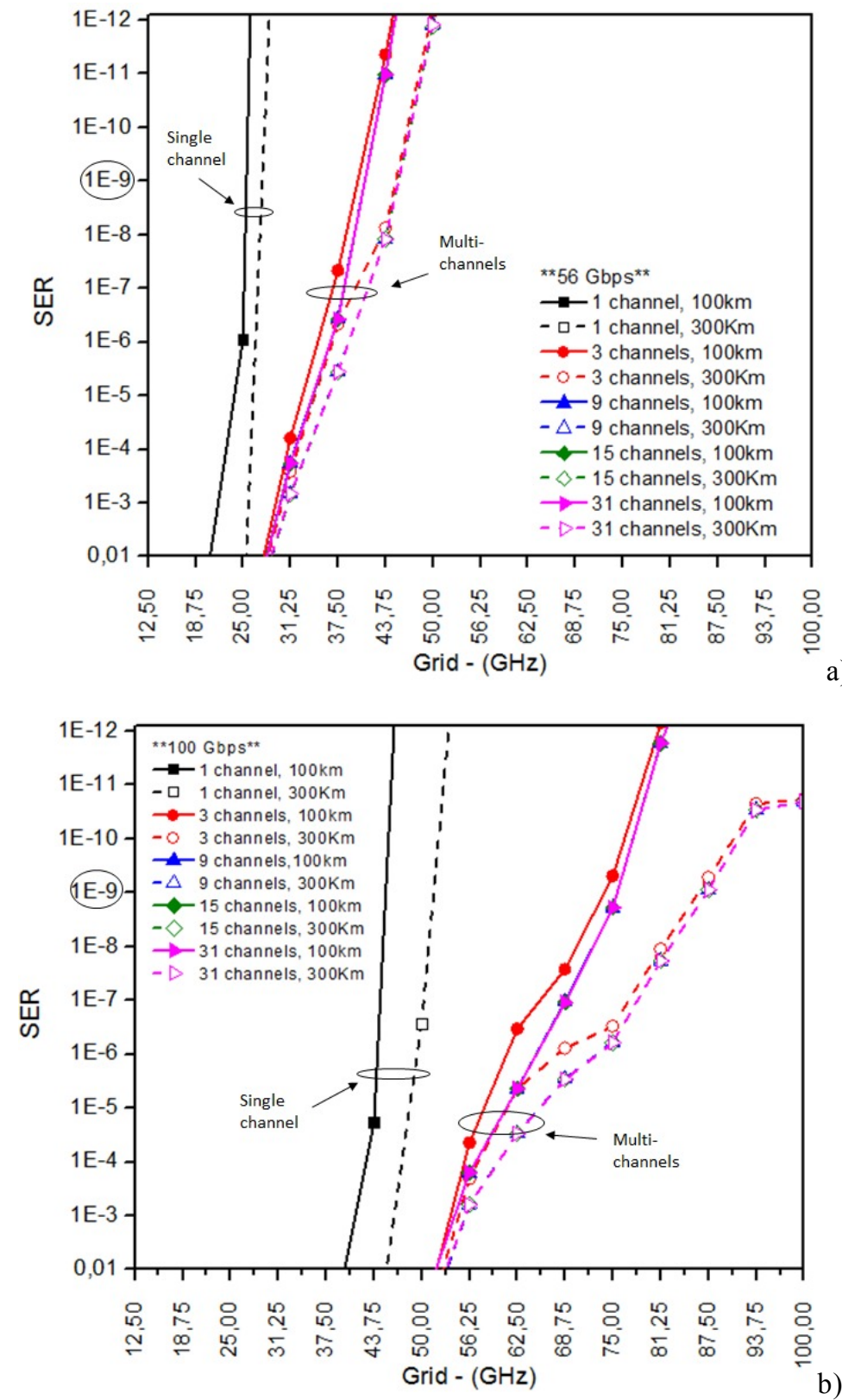

Figure 3: Fiber link of 100 and $300 \mathrm{Km}$ - QPSK (a) 56Gbps, (b) $100 \mathrm{Gbps}$.

\section{ACKNOWLEDGMENTS}

This work was developed at the Pontifical Catholic University of Campinas (PUC-Campinas)-CEATEC, in cooperation with the Photonics Technology Lab (LTF), at Unicamp School of Electrical Engineering FEEC.

\section{REFERENCES}

[1] Cisco, "White paper: Cisco VNI Forecast and Methodology, 2015- 2020," White Paper, Jun. 2016. [Online]. Available: http://www.cisco. com/c/en/us/solutions/collateral/service-provider/visual-networkingindexvni/mobile-white-paper-c11-520862.html 
[2] R. Ramaswami and K. N. Sivarajan. Optical Networks: A Practical Perspective. Morgan Kaufmann, 3rd edition, 2010.

[3] Stavdas A. et al. Taking Advantage of Elastic Optical Networks. In: López V., Velasco L. (eds) Elastic Optical Networks. Optical Networks. Springer, Cham, 2016.

[4] ITU-T G694.1, "Spectral grids for WDM applications: DWDM frequency grid" ;

[5] S. Azodolmoky, M. Klinlowski, E. Marin, D. Careglio, J. S. Pareta, I. Tomkos, “A survey on physical layer impairments aware routing and wavelength assignment algorithms in optical networks, Journal Computer Networks: The International Journal of Computer and Telecommunications Networking, Vol. 53, pp. 926-944, (2009).

[6] Nag, A.; Tornatore, M.; Mukherjee, B., "Optical Network Design With Mixed Line Rates and Multiple Modulation Formats," Lightwave Technology, Journal of, vol.28, no.4, pp.466-475, Feb.15, 2010

[7] Gerstel, O.; Jinno, M.; Lord, A.; Yoo, S.J.B , "Elastic optical networking: a new dawn for the optical layer?," Communications Magazine, IEEE, vol.50, no.2, pp.s 12-s20, February 2012

[8] I. Tomkos, S. Azodolmolky, J. Solé-Pareta, D. Careglio and E. Palkopoulou, "A tutorial on the flexible optical networking paradigm: State of the art, trends, and research challenges," in Proceeds. IEEE, vol. 102, no. 9, p. 1317, Sept. 2014.

[9] I. B. Martins, G Castañón, F. Lezama and I. Aldaya, "Impact of spectral width on signal transmission distance in elastic optical network," 2014 16th International Conference on Transparent Optical Networks (ICTON), pp. 1-3.; Graz, 2014

[10] Lathi, B. P. Modern digital and analog communication systems. 3rd ed. New York: Oxford University, 1998. 